\title{
A Prototype of Wireless Power and Data Acquisition System for Large Detectors
}

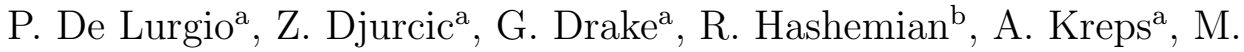

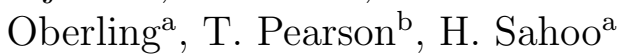 \\ ${ }^{a}$ Argonne National Laboratory, Argonne, IL 60439, USA \\ ${ }^{b}$ Northern Illinois University, Dekalb, IL 60115, USA
}

\begin{abstract}
We have developed a prototype detector and data acquisition module that incorporates wireless power and wireless data transmission techniques. The module has no electrical connections. It receives power using photovoltaic devices, and communicates control, timing, trigger, and data using the $802.11 \mathrm{n}$ wireless communication standard. The work is part of a study for building a large detector having many readout channels, where it is desirable to reduce the cable plant and infrastructure. The system could also be deployed in smaller detectors that require mobility or are difficult to cable due to extreme conditions. We describe the design and operation of the prototype module, including benchmark performance measurements, and discuss aspect and issues in extrapolating to a large detector system.
\end{abstract}

Keywords: Wireless communications, RF, Optical

Email address: zdjurcic@anl.gov (Z. Djurcic) 
1

2

\section{Introduction}

The goal of this R\&D project was to design and build a detector module that operates from wireless power and transmits data using wireless data transmission techniques. The motivation is the elimination of the massive cable plants that are typical of large detectors, thereby reducing detector dead mass, simplifying installation and repair, and possibly reducing cost. In particular, cabling can create technical challenges for detectors in remote locations or hostile environments. We used this work to study the feasibility of building a large detector that would be constructed as an array of single detector modules. The technologies that we used in this work are not necessarily optimal for all detectors and experiments. Rather, our goal was to choose technologies that could demonstrate the concept as a basis for thinking about the challenges and issues in building a large detector using these techniques. One of the motivations in this work was consideration of building a read-out system for a large neutrino detector such as a water-Cherenkov detector configured with photomultiplier tubes (PMT). The readout rate of these detectors is often dominated by the dark noise rate, which can be of order $10 \mathrm{KHz}$ [1]. Examples of such detectors include water-based LBNE [2], MEMPHYS [3], Hyper-K [4], and CHIPS [5] detectors. Detectors used for homeland security also use similar technologies and techniques. Simplifying the implementation of large detectors with high channel counts could facilitate detection of nuclear materials and other contra-band.

Other applications could benefit from infrastructure simplification, increased mobility, and stand-off distance capability of wireless techniques. Some examples of these include monitoring of the neutrino flux at a nuclear 
reactor, large mobile neutron detectors for detection of radioactive material in security applications, and detectors operating in high-radiation areas. Operation in real world conditions sometimes requires the ability for detectors to be mobile, and/or the ability to operate continuously for long periods of time, both of which can benefit from using low-power wireless techniques.

For neutrino and other low-rate experiments, the single photoelectron rate often dominates the event rate. If we assume for each event that the data would be comprised of 6 bytes, 2 bytes of pulse height information and a 4 byte time-stamp, at $10 \mathrm{kHz}$, this translates to $60 \mathrm{kBytes}(\mathrm{kB})$, or $480 \mathrm{kbits} / \mathrm{second}(\mathrm{kb} / \mathrm{s})$. For a single detector element, these data rates are achievable with a wireless readout. Extrapolating to a detector with a large number of channels (for example, 40000 PMTs are considered in some variations of a water-based LBNE [2]) translates into a data rate (on the order of $\sim 20 \mathrm{~Gb} / \mathrm{s}$ in the LBNE example), which is one of the challenges for the wireless readout.

Successful demonstration of an all wireless system could be transformational when realized in a practical, cost effective, and reliable fashion. We intend to use this prototyping work as a starting point for using these principles in new read-out technologies.

\section{Design Considerations}

For wireless data transmission, we considered two techniques: radiofrequency (RF) wireless and free-space optical data transfer. These will be discussed in more details. For the wireless power transfer, we considered RF and optical methods. There are other approaches for transmitting the 
Table 1: Target specifications for the wireless data acquisition system.

\begin{tabular}{c|c}
\hline \hline Specification & Target \\
\hline Maximum event rate (single p.e.) & $10 \mathrm{kHz}$ \\
\hline Bytes per event & $6(2$ pulse height, 4 time-stamp $)$ \\
\hline Average data rate per front-end channel & $60 \mathrm{kB} / \mathrm{s}$ \\
\hline Total power consumption @ $10 \mathrm{kHz}$ & $250 \mathrm{~mW}$ \\
Digital & $120 \mathrm{~mW}$ \\
Front-end & $30 \mathrm{~mW}$ \\
HV & $100 \mathrm{~mW}$ \\
\hline Data transfer rate & $35 \mathrm{Mb} / \mathrm{s}$ \\
\hline Bit error rate & $<1 \times 10^{-12}$ \\
\hline Additional Features & Self-trigger for pedestals \\
& Data pull \\
\hline \hline
\end{tabular}

power wirelessly such as strongly coupled magnetic resonance techniques [6].

Wireless technologies have been studied for a wide variety of applications; for example in space and radiation research [7], radioactive material sensor network [8] and in the field of radiation detection [9]. In selecting technologies, a preference was placed on the use of inexpensive, off-the-shelf technologies that could be implemented relatively easily, with minimal risk, and which could meet the performance goals of this project. The initial target specifications for the prototype system are summarized in Table 1. The parameters can generally be obtained using off-the-shelf solutions. Our estimates using 
existing hardware $[10,11,12,13,14]$ are $\sim 100 \mathrm{~mW}$ for the high-voltage generation of the PMT, $\sim 120 \mathrm{~mW}$ for the data processing and wireless data transmission, and $\sim 30 \mathrm{~mW}$ for the front-end needed for processing and digitization of the PMT signal.

\subsection{Wireless Data Transmission}

For wireless data transmission, optical links generally support higher data rates compared with RF. Individual free space links over distances of meters can achieve transfer rates of approximately $1 \mathrm{~Gb} / \mathrm{s}$ [15]. However, RF transmission does not require line-of-sight, and an individual transmitter can communicate with many front-end receivers. RF data transmission was chosen for this project for these reasons. An issue with RF is that the bandwidth of an individual link is typically modest, approximately $100 \mathrm{MB} / \mathrm{s}$, and the available RF bandwidth is shared by all transmitting elements of the detector.

There are two primary categories of commercial wireless data technologies: mobile/cellular [16] and wireless local area network (WLAN) [10]. We found that cellular networks, including the latest $4 \mathrm{G}$ technologies, offer higher distance ranges than WLAN technologies, but this comes at the expense of a lower overall data rate [17]. For this project, we focused on WLAN technologies, since they have the highest data throughput, which is the rate of successful message delivery over a given communication channel. We selected the $802.11 \mathrm{n}$ protocol, which at the time, offered the highest data throughput of $65 \mathrm{Mb} / \mathrm{s}$ [11]. The available payload data rate, which is the maximum available data throughput after accounting for the error correction overhead is $\sim 35 \mathrm{Mb} / \mathrm{s}$. This was sufficient for our single prototype front-end. 
The 802.11n wireless network operates in both the $2.4 \mathrm{GHz}$ and $5 \mathrm{GHz}$ frequency bands. We utilized the $5 \mathrm{GHz}$ band in our study, which has an overall bandwidth of approximately $1.2 \mathrm{GHz}$ from about $4.9 \mathrm{GHz}$ to $6.1 \mathrm{GHz}$. This ignores limitations imposed by the governing agencies that dictate the use of the electromagnetic spectrum. Under certain circumstances, the full spectrum can be available for use with devices capable of dynamic frequency selection [12]. The approach is needed in order to implement the use of a large number of access points in a large detector system to accommodate the total payload data rate needed by the data acquisition system. Single stream 802.11n access points can have an individual operating bandwidth of $20 \mathrm{MHz}$ or $40 \mathrm{MHz}$. With $20 \mathrm{MHz}$ wide channels, it should be possible to populate the $1.2 \mathrm{GHz}$ wide frequency band with up to 48 access points. For a single stream $802.11 \mathrm{n}$ link this translates into a total payload data rate of $1.68 \mathrm{~Gb} / \mathrm{s}$, as illustrated in Fig. 1. The system consists of many access points ( 48) each communicating to many clients $(\sim 64)$, for a total of 3072 clients. This assumes $480 \mathrm{~kb} / \mathrm{s}$ per front-end and a transfer rate of $35 \mathrm{Mb} / \mathrm{s}$.

For the detectors with a large number of channels, the available payload data rate is insufficient. There are two potential extensions of this concept that might result in an increased data throughput. The first is the use of directional antennas and intrinsic shielding created by the detector volume to allow the same frequency range to be re-used in different locations on the detector. The second approach is to include extensions of the standard $802.11 \mathrm{n}$ protocol that allow for multiple-input and multiple-output (MIMO) [13] wireless streams, which could significantly increase data throughput. For example, $4 \times 4$ MIMO requires $40 \mathrm{MHz}$ wide channels, which means the $1.2 \mathrm{GHz}$ 


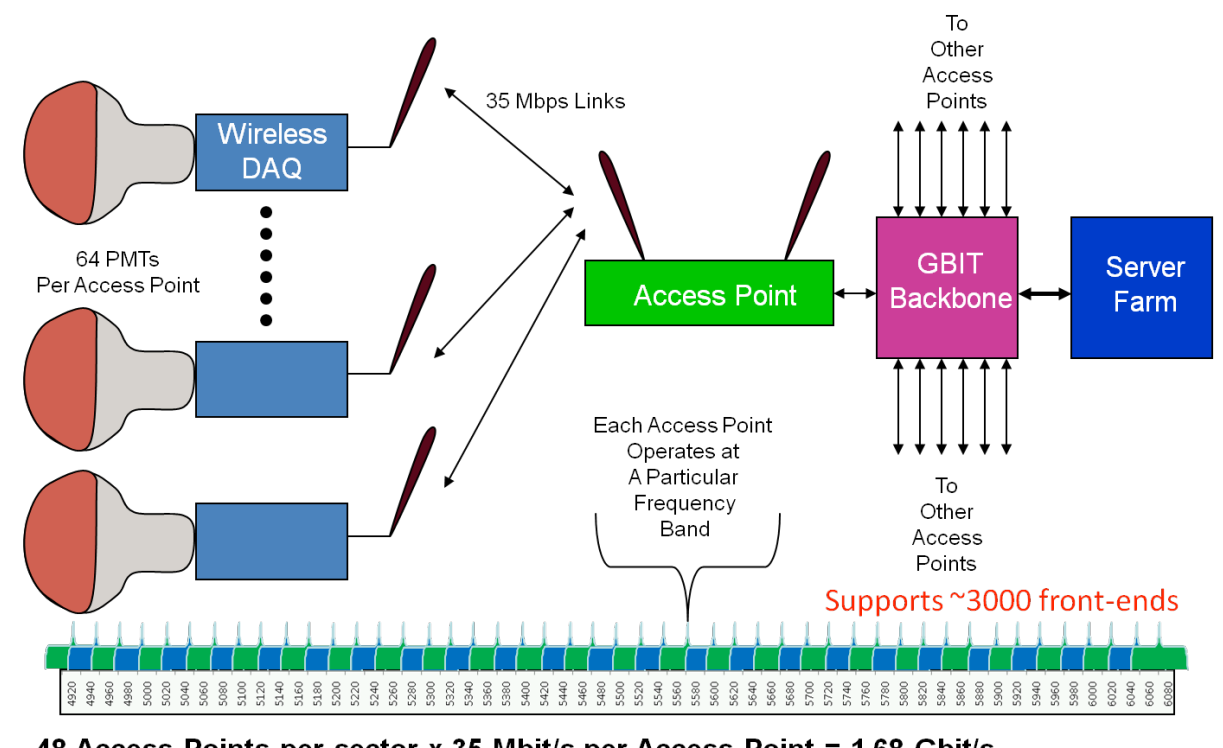

- 48 Access Points per sector x 35 Mbit/s per Access Point $=1.68 \mathrm{Gbit} / \mathrm{s}$

Figure 1: Allocation of frequency space for wireless data transmission.

frequency band would support approximately 24 access points. The $4 \times 4$ MIMO implementation would then support 4 simultaneous transmit and receive streams to yield a data throughput of $600 \mathrm{Mb} / \mathrm{s}$, which translates into a maximum payload data rate of $320 \mathrm{Mb} / \mathrm{s}$. The total "sector" payload data rate would then be $7.68 \mathrm{~Gb} / \mathrm{s}$.

The combination of these two extensions to the multi-access point configuration could provide much higher data throughput to read-out a detector with large channel count. Note that the above estimates do not account for latency in the communication. In practice, the latencies will impact the achievable throughput. In a real system, the DAQ system would probably have to "pull" data from the front-ends. This requires a handshake between client and access point and increases latencies slightly. However, this controls the readout process and avoids the need for clients requesting to send data. 
For example, the server might request data from each front-ends once per second, provided that there is adequate data storage in the front-ends, and that the event rates are modest.

\subsection{Wireless Power Transmission}

We considered and tested both optical and RF power transfer methods. The optical demonstrator utilizes a high-power light-emitting diode (LED) that was collimated into an 8 inch diameter beam. The light was received by a photovoltaic (PV) panel, as shown in Fig. 2. We used an OSRAM SFH $4751 \mathrm{LED}$ with $3.5 \mathrm{~W}$ optical output. The LED wavelength is $940 \mathrm{~nm}$, which matches the peak efficiency of the Delsolar $156 \times 156 \mathrm{~mm}^{2}$ photovoltaic cell used in our $312 \times 280 \mathrm{~mm}^{2} \mathrm{PV}$ panel array. Figure 3 shows the power received from this system as function of transmission distance. The performance met our prototype requirements of receiving $0.25 \mathrm{~W}$ power at 5 meters. In addition to the studies conducted in our lab, we also performed a test with the solar panel array exposed to the sun light. We found that it was possible to receive more than $0.25 \mathrm{~W}$ to the prototype front-end, under bright conditions.

The RF power transfer test consisted of a function generator driving a $14 \mathrm{dBi}$ gain Yagi antenna at $915 \mathrm{MHz}$ with an output power of $10 \mathrm{dBm}$. The receiver used an $11 \mathrm{dBi}$ gain patch antenna, as shown in Fig. 4. The measurements were taken inside a large room, and the apparatus was placed at $\sim 2 \mathrm{~m}$ from the floor to reduce the scattering from surrounding objects. The data from these tests, as a function of transmission distance, are shown in Fig. 5. The data points are in good agreement with the expectation from the Friis transmission equation [18], which relates the ratio of power received 


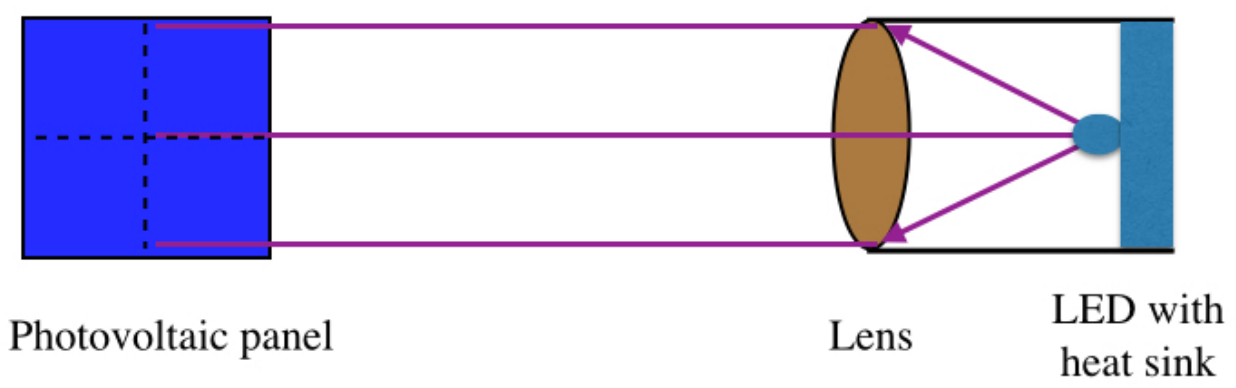

Figure 2: Apparatus for optical power transmission. The photovoltaic receiver (left), the LED and lens are inside a tube (right).

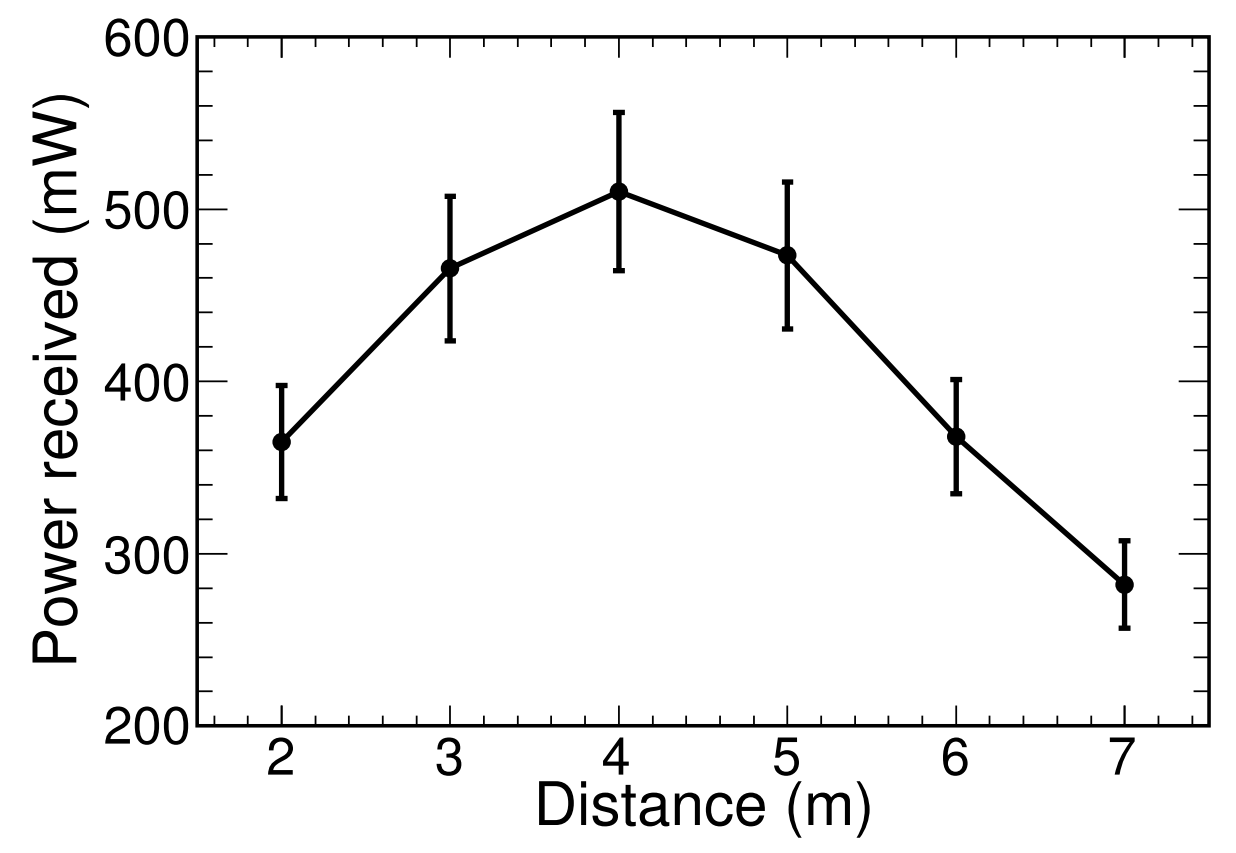

Figure 3: Power received $(\mathrm{mW})$ by the photovoltaic panel as a function of distance $(\mathrm{m})$ from the optical source. The optical source used was a $3.5 \mathrm{~W}$ LED with a $940 \mathrm{~nm}$ wavelength and a focusing lens. 


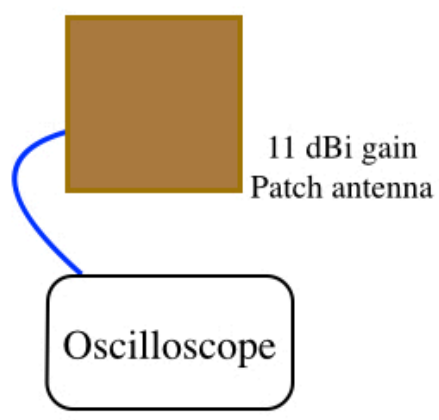

$P_{r}$ to power transmitted $P_{t}$ for a given pair of antennas with gains $G_{t}$ and $G_{r}$, operating at an wavelength $\lambda$, and separated by a distance $R$, as given in Eq. 1. There is a rapid fall off of received power as the transmission distance increases. At 5 meters the power loss is $20 \mathrm{dBm}$, which requires a $25 \mathrm{~W}$ source to receive $250 \mathrm{~mW}$, our target power.

$$
\frac{P_{r}}{P_{t}}=G_{t} G_{r}\left(\frac{\lambda}{4 \pi R}\right)^{2}
$$

For our prototype device, the optical system was the chosen approach. The

Figure 4: Apparatus for RF power transmission. The RF transmitter (14 dBi gain Yagi antenna) with a function generator is in the right, the $\mathrm{RF}$ receiver (11 dBi gain patch antenna) with a oscilloscope is in the left.

152

power source and receiver were relatively easy to implement. RF power transmission requires converting the RF power into a DC supply, which is commercially available but typically limited to $\sim 100 \mathrm{~mW}$ of received power [14]. The optical power transfer technique met the power requirements for this demonstrator. RF power could be a practical choice for a large detector, 


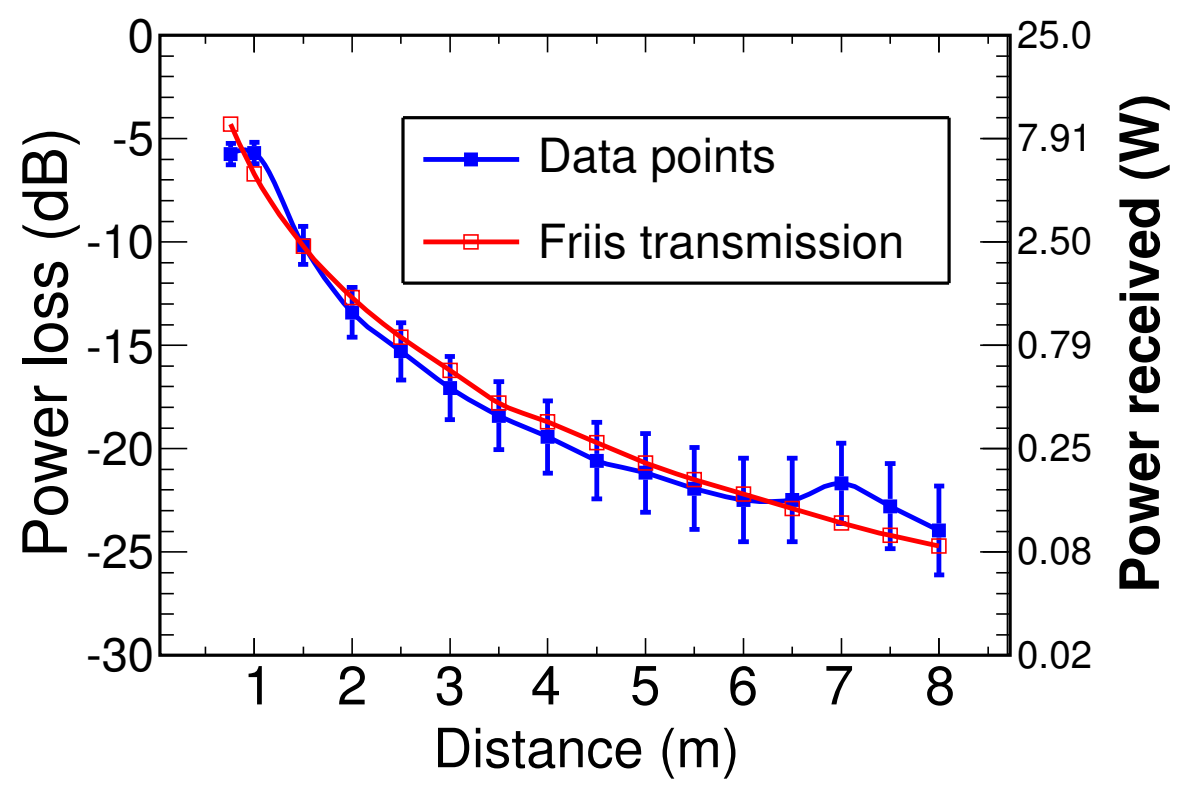

Figure 5: Power loss $(\mathrm{dB})$ in the RF power transmission test as a function of distance (m) from the source. Solid dots with error bars are the measurements and open dots are the expectation from the Friis transmission equation. The source is a $915 \mathrm{MHz} 10 \mathrm{dBm}$ transmitted through a $14 \mathrm{dBi}$ gain Yagi antenna and received by an $11 \mathrm{dBi}$ gain patch antenna. The scale on the right shows the power that would be received for a $25 \mathrm{~W}$ (44 $\mathrm{dBm})$ source.

since one source can power many transmitters. This could potentially reduce the complexity and cost of the system. For example, at 5 meters the same power $\pm 3 \mathrm{dBm}$ is being transmitted into a $2 \times 2 \mathrm{~m}^{2}$ area. For a $50 \mathrm{~W}$ output, a single equivalent source could power as many as 40 front-ends depending on the packing density. One potential issue to be addressed in a future studies when using the RF power transfer techniques is electromagnetic interference from the RF signal. 

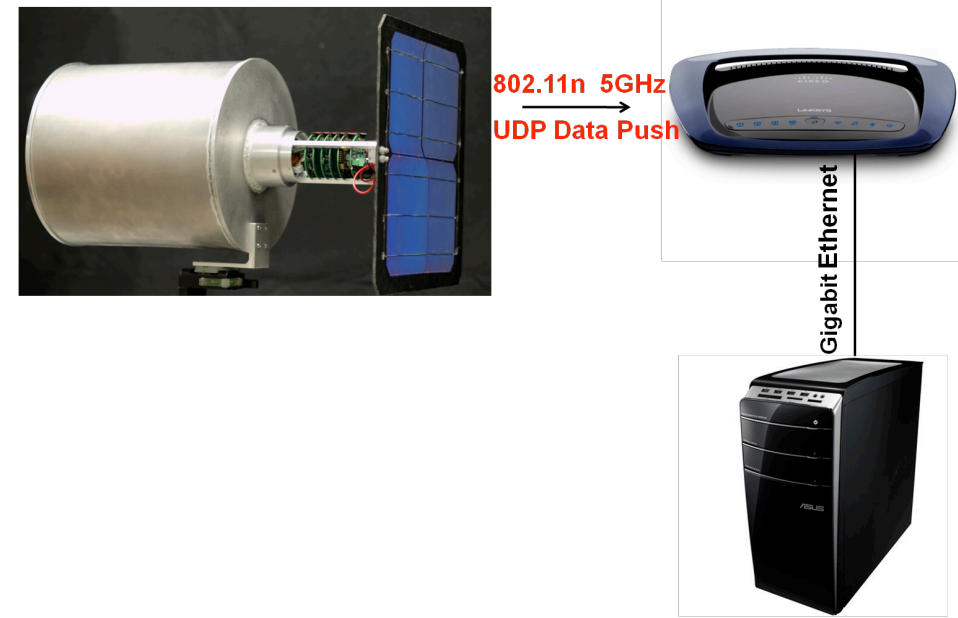

PC with Scientific Linux

Figure 6: Configuration of the prototype readout system. The front-end (left) transmits data wirelessly and collected by the access point (upper right) and stored in PC with scientic linux (lower right). 

(left), and the complete system with PMT is shown in Fig. 8 (right).

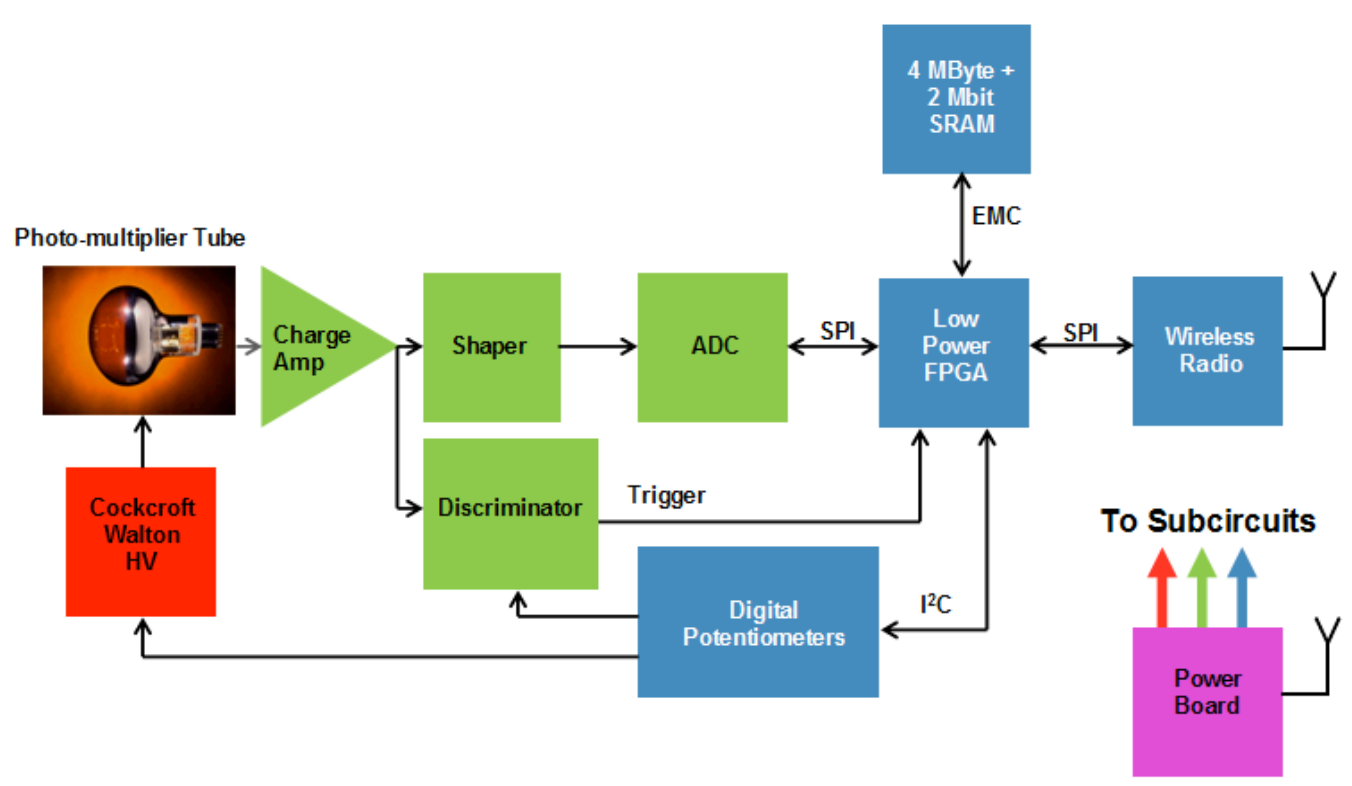

Figure 7: Block diagram of the wireless prototype system.

185

\subsection{Hardware Description}

Figure 7 shows a block diagram of the wireless demonstrator. The prototype system is comprised of 4 boards that include: a board for generating high voltage for the PMT; a front-end board for shaping and digitization of the PMT signal; a digital board for processing the data and wireless data transmission; and a power board for receiving wireless power and generating the different voltages needed by the system. The system was designed to connect to a photovoltaic panel and send data wirelessly using $802.11 \mathrm{n}$ in the $5 \mathrm{GHz}$ band. The four boards in the PMT base are shown in Fig. 8

The power board uses a LTC 3105 [21] switch mode step-up converter to convert the $1.85 \mathrm{~V}$ received from the photovoltaic panel into $5 \mathrm{~V}$, which 


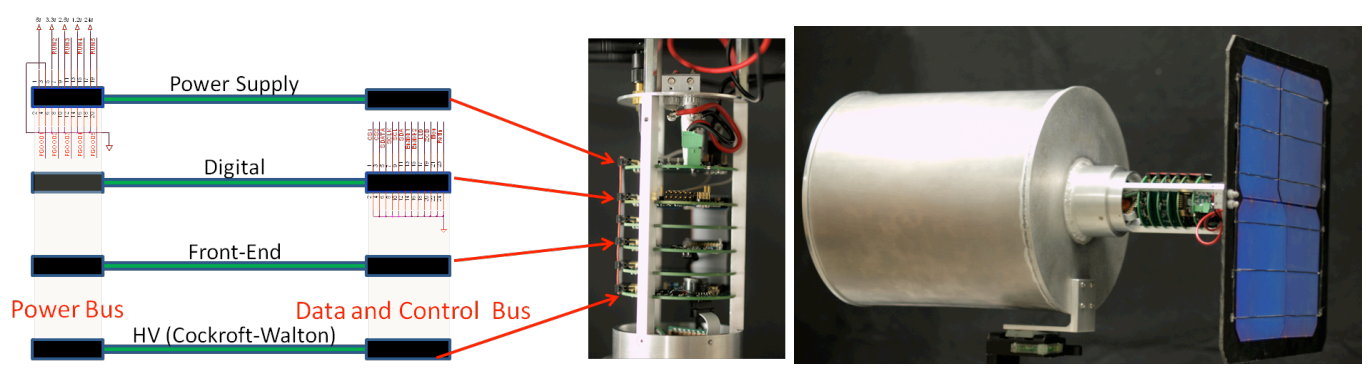

Figure 8: Left: Four printed circuit boards of the wireless prototype system assembled within the PMT base. The boards are power board, digital board, front-end board, and the high-voltage board. Right: Picture of the completed system shown with light tight PMT enclosure and photovoltaic panel attached to PMT base.

is used as the system voltage. The energy is stored in a series of ultracapacitors [22] with an effective total capacitance of $2.5 \mathrm{~F}$. We used stepdown convertors [23] and step-up converters [24] to convert $5 \mathrm{~V}$ into voltages needed to run the system components.

The digital board hosts the processor, wireless radio, and additional digital integrated circuitry (i.e. external low-power Static Random-Access Memory (SRAM) and Serial Peripheral Interface (SPI) flash [25]). The wireless radio used was a cBOWL221a [11] commercial board. It uses $802.11 \mathrm{n}$ single stream communication and has an SPI interface. This module was chosen because of its low power consumption. The radio incorporates all of the 802.11n protocol, with the Transmission Control Protocol (TCP)/IP or UDP/IP stack incorporated inside the processor. The processor used was a Microsemi A2F200 Smart Fusion [26]. The Field Programmable Gate Array (FPGA) fabric is used for the discriminator logic, Analog-to-Digital Converter (ADC) readout, time-stamping, and event word generation. It directly accesses the external memory and stores the events using the same memory 
controller as used by the processor.

The high voltage board uses a Cockroft-Walton (CW) switching circuit to boost the $24 \mathrm{~V}$ input voltage up to $2000 \mathrm{~V}$. This also operates from wireless power, and the noise performance was sufficient to measure single photoelectrons.

The front-end board includes a charge-sensitive preamplifier, shaping amplifier, programmable discriminators, and a 12-bit ADC. The output of the charge sensitive amplifier connects to both a uni-polar and a bi- polar shaping amplifier. The uni-polar shaping amplifier output is digitized by an AD7451 ADC [27] for measuring the pulse-height. The bi-polar shaping amplifier is used as the input to a pair of comparators, one of which is used for pulseheight discrimination and the other for timing. The comparator outputs connect to the A2F200 FPGA fabric (digital board) over the data and control bus. As mentioned earlier, the A2F200 FPGA fabric is used for the discriminator logic to control the ADC readout.

\subsection{Performance Discussion}

The prototype system has been built and tested. It was powered wirelessly using the optical source, which was received by the photovoltaic panel. Tests were performed with the system running from wireless power and by sending and receiving the data wirelessly. The performance achieved is summarized in Table 2.

As shown in the Table 2, the power consumption of the system is 386 $\mathrm{mW}$, higher than the initial $250 \mathrm{~mW}$ target. The primary reasons for the increase are higher power consumption in the digital and the high-voltage boards. In the case of the digital board, the target specification was based 
Table 2: Summary of initial goals and achieved performance for the wireless data acquisi-

\begin{tabular}{c|c|c} 
tion system. & Target & Performance \\
\hline \hline Specification & $250 \mathrm{~mW}$ & $386 \mathrm{~mW}$ \\
Dotal power consumption (@ $10 \mathrm{kHz})$ & $120 \mathrm{~mW}$ & $216 \mathrm{~mW}$ \\
Front-end & $30 \mathrm{~mW}$ & $39 \mathrm{~mW}$ \\
HV & $100 \mathrm{~mW}$ & $131 \mathrm{~mW}$ \\
\hline Maximum event rate & $10 \mathrm{kHz}$ & $80 \mathrm{kHz}$ \\
\hline Data transfer rate & $35 \mathrm{Mb} / \mathrm{s}$ & $11 \mathrm{Mb} / \mathrm{s}$ \\
\hline Bit Error Rate & $<1 \times 10^{-12}$ & Dropped Packets \\
\hline \hline
\end{tabular}

on Microsemi spreadsheet power estimator, which appears to underestimate actual operation. The high-voltage board used a resistive divider in the PMT, rather than direct connection of dynodes to the CW supply. This caused the power consumption to increase. We believe that the original power consumption goal can be achieved given that both of these problems are understood, although noise from the CW coupling into the dynodes would have to be addressed.

The system is capable of sending greater than $10 \mathrm{k}$ events $/ \mathrm{s}(60 \mathrm{kB} / \mathrm{s})$. However, the critical parameter for performance in a large detector is the maximum data transfer rate. The specification of $35 \mathrm{Mb} / \mathrm{s}$ is the maximum transfer rate of an 802.11n single stream link, and this was our target. While this is achievable with the radio used here, it does necessitate zero SPI bus latency and a $50 \mathrm{MHz}$ SPI clock. The maximum transfer rate that we achieved was $11 \mathrm{Mb} / \mathrm{s}$. The reason for this was twofold. First, the maximum 
SPI clock rate in the A2F200 is set by the master clock divided by 4 . This limits the maximum SPI clock rate to $25 \mathrm{MHz}$. Second, latencies caused by FreeRTOS [28], the driver code, and the speed of the A2F200 processor, account for the remaining difference. The pin-compatible A2F500 supports a $50 \mathrm{MHz}$ SPI clock and will be implemented in the future. This simple change should yield $18 \mathrm{Mb} / \mathrm{s}$. Reducing the latency would require the elimination of FreeRTOS and driver optimization.

Our target bit error rate (BER) was less than $1 \times 10^{-12}$. A bit error rate test (BERT) program was written to analyze the performance. The prototype system was run continuously over 100 hours with no bit errors observed in any received packets. This is a result of operation of a relatively simple system with one broadcast source at a time, in the largely unused $5 \mathrm{GHz}$ band in our lab. It is also due to the forward error correction that is incorporated in the $802.11 \mathrm{n}$ protocol. However, significant numbers of dropped packets were observed, on the order of 1 in 2000. It is believed that this is due to the use of UDP. While more efficient than TCP, UDP does not have retransmission capabilities. We traced the problem to our access point, having observed the drop packets with the same frequency by using a computer to generate fake data. The nature of UDP will require some consideration of error detection and recovery for a large system. It may be possible to significantly reduce the systematic packet loss by using a protocol such as the Pretty Good Protocol (PGP) [29], for dropped-packet detection and retransmission mechanism.

A few results of data collected with the prototype system operated from wireless power and with wireless data readout are shown in Fig. 9. The 

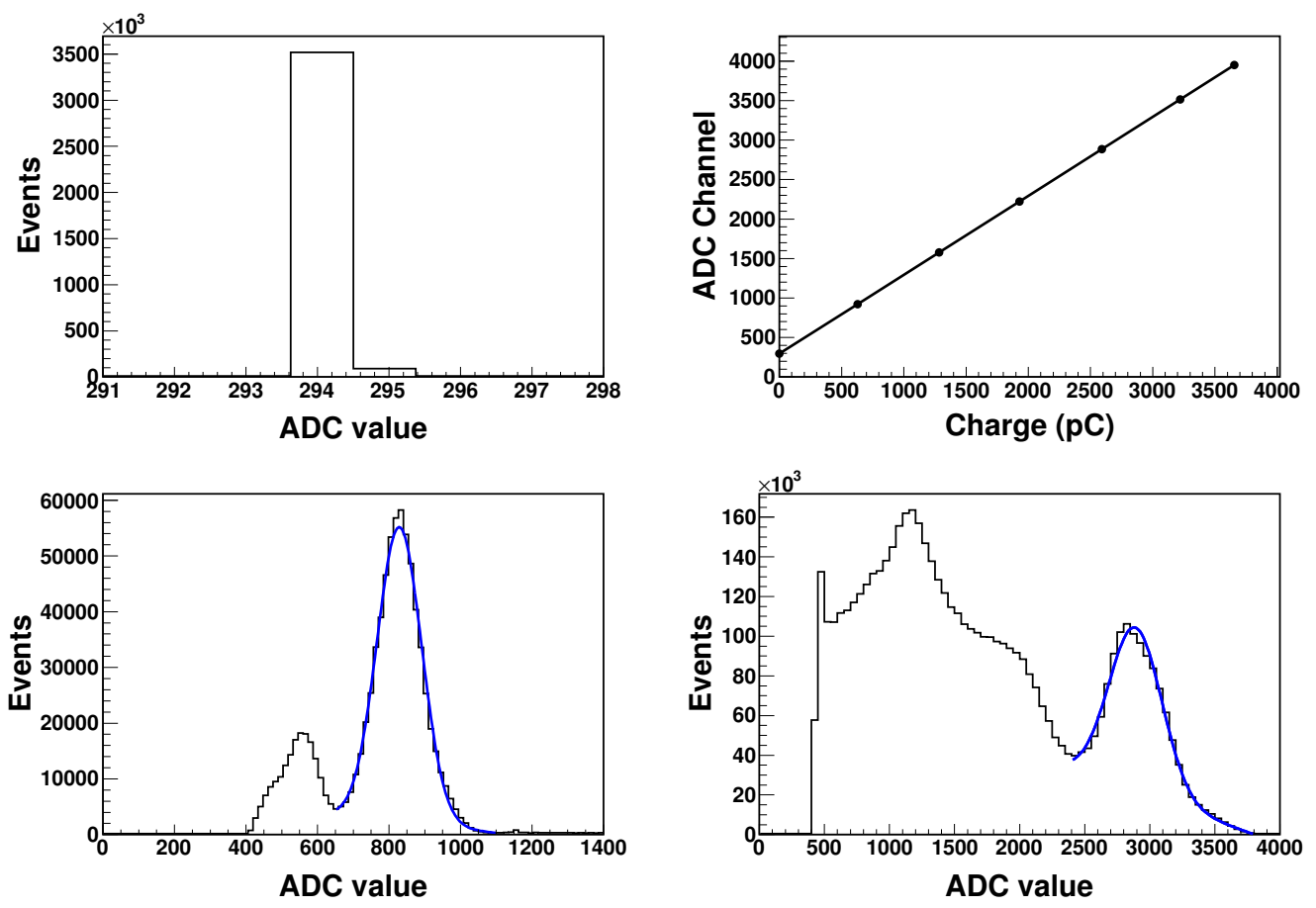

Figure 9: Upper Left: Measurement of pedestal. The system is operated with conditions 10k events/s, self-triggered, HV turned on with no PMT, system powered wirelessly and wireless readout. Upper Right: Response from electronic charge injection. The system is operated with conditions self-triggered, PMT HV on, system powered wirelessly and wireless readout. Lower Left: Response from $60 \mathrm{keV}^{241} \mathrm{Am}$ source. The system is operated with conditions $1 \mathrm{k}$ events/s, self-triggered, PMT HV 1300V, system powered wirelessly and wireless readout. Lower Right: Response from $661.7 \mathrm{keV}{ }^{137} \mathrm{Cs}$ source. The system is operated with conditions 250 events/s, self-triggered, PMT HV $1200 \mathrm{~V}$, system powered wirelessly and wireless readout.

front-end circuit exhibits very low noise as demonstrated with the pedestal data. The integral linearity over the full digitization range shown in Fig. 9 (upper right) is better than $0.4 \%$. To test remote data acquisition capability, a sodium iodide crystal was attached to the PMT and tested with ${ }^{241} \mathrm{Am}$ 
272

(Fig. 9, lower left) and ${ }^{137} \mathrm{Cs}$ (Fig. 9, lower right) sources.

\section{Summary and Future Prospects}

We successfully designed and built a wireless data acquisition system implemented in a photomultiplier tube base that operates from wireless power and sends data wirelessly. The power consumption, while slightly greater than our target, is still low enough to allow the system to operate from our optical power transfer system. Future improvements would include improving the SPI clock rate by using the A2F500, eliminating FreeRTOS, and reducing the SPI bus latency. This will reduce power consumption in the digital board. The high-voltage board should be redesigned to integrate correctly into the chosen PMT. In the longer-term, RF power transfer should be explored to facilitate the simplification of using one transmitter to power many receivers. Investigating the use of a custom application-specific integrated circuits for low-power operation of the front-end and Cockroft-Walton control circuitry might also be considered for a larger system.

The use of the pin-compatible A2F500 SmartFusion device should increase the transfer rate from $11 \mathrm{Mb} / \mathrm{s}$ to $18 \mathrm{Mb} / \mathrm{s}$. Assuming $480 \mathrm{~Kb} / \mathrm{s}$ average data rate from our front-ends and assuming a conservative estimate of the latency between request for data and transmission of data of $10 \mathrm{~ms}$, the burst transfer rate achieved would support 18 and 27 front-ends for 11 and $18 \mathrm{Mb} / \mathrm{s}$ respectively. A system with 48 access points would support 1296 front-ends. 


\section{Acknowledgments}

We acknowledge the support of both Argonne National Laboratory and Office of High Energy Physics in the Office of Science at the Department of Energy for this project. Argonne National Laboratory's work was supported by U.S. Department of Energy under contract DE-AC02-06CH11357. We would also like to thank Jeff Maus and Henric Lindn of ConnectBlue for supporting our efforts to incorporate the c221a 802.11n wireless radio working in our system. We especially thank Greg Mears of the Microsemi Corporation for his help with the SmatFusion system-on-chip device.

\section{References}

[1] R7081-02 Datasheet, Nov. 12. 2003, Hamamatsu Photonics K.K., 3256, Sunayama-cho, Naka-ku, Hamamatsu City, Shizuoka Pref., 430-8587, Japan.

[2] T. Akiri et al., The 2010 Interim Report of the Long-Baseline Neutrino Experiment Collaboration Physics Working Groups, arXiv: 1110.6249.

[3] D. Autiero et al., Large underground, liquid based detectors for astro-particle physics in Europe: scientific case and prospects, arXiv: 0705.0116 .

[4] K. Abe et al., Letter of Intent: The Hyper-Kamiokande ExperimentDetector Design and Physics Potential, arXiv:1109.3262.

[5] P. Adamson et al., CHerenkov detectors in mine PitS (CHIPS): Letter of Intent to FNAL, arXiv:1307.5918. 
[6] A. Kurs, et al., Science 317, 83 (2007).

[7] A. Gabrielli, et al., http://dx.doi.org/10.1016/j.nima.2014.03.065.

[8] D. Katsis, et al., Nucl. Instr. meth. A 652 (2011) 94-98, http://dx.doi.org/10.1016/j.nima.2010.08.121.

[9] E. M. Becker, et al., Nucl. Instr. Meth. A 761 (2014) 99-104, http://dx.doi.org/10.1016/j.nima.2014.05.096.

[10] S. Baek and B. D. Choi, Performance analysis of power save mode in IEEE 802.11 infrastructure WLAN, in Telecommunications, 2008. ICT 2008. Intl Conf., Jun 2008, pp. 1-4.

[11] cBOWL221a Product Brief, cBProduct-0811-01 (1.6), Aug. 2011, ConnectBlue AB, Norra Vallgatan 64 3V, SE-211 22 Malmö, Sweden.

[12] Part 11: Wireless Lan Medium Access Control (MAC) and Physical Layer (PHY) Specifications, IEEE Standard 802.11 ${ }^{\mathrm{TM}}-2012$.

[13] Ohlmer E., Fettweis G., Plettemeier D., "MIMO system design and field tests for terminals with confined space - impact on automotive communication," Antennas and Propagation (EUCAP), Proceedings of the 5th European Conference, pp.2886-2890, 11-15 April 2011.

[14] P1110 915 MHz RF Powerharvester ${ }^{\mathrm{TM}}$ Receiver Datasheet, Rev A, Apr. 2010, Powercast Corporation, 566 Alpha Drive, Pitsburgh, PA 15238, United States. 
[15] P. De Lurgio, W. Fernando, B. Salvachua, R. Stanek, D. Underwood, D. Lopez, "New Optical Link Technologies for HEP Experiments", in Proc. DPF-2011 Conference, Providence RI, August 8-13, 2011.

[16] H. Balakrishnan, S. Seshan, and R. H. Katz, Improving reliable transport and handoff performance in cellular wireless networks, ACM Wireless Networks , vol. 1, no. 4, 1995.

[17] S. Grech, "Game Analysis: Cellular vs WLAN", S-38.042, Networking Laboratory, Helsinki University of Technology.

[18] H. T. Friis, "A Note on a Simple Transmission Formula", Proceedings of the IRE., vol. 34, no. 5, pp. 254 - 256, May 1946.

[19] ScientificLinux, Available: http://www.scientificlinx.org.

[20] M. Petrovic, M. Aboelaze, "Performance of TCP/UDP under Ad Hoc IEEE802.11", http://arxiv.org/pdf/cs/0311049v1.pdf.

[21] LTC3105 Datasheet, 3105fa Rev A, Feb. 2011, Linear Technology Corporation, 1630 McCarthy Blvd., Milpitas, CA 95035, United States.

[22] ESHSR-0010C0-002R7 Datasheet, (446-901) 750-8, Gomae-dong, Giheung-gu, Yongin-si, Gyeonggi-do, Korea.

[23] LT3104 Datasheet, 3104f, Oct. 2011, Linear Technology Corporation, 1630 McCarthy Blvd., Milpitas, CA 95035, United States.

[24] LT3473 Datasheet, 3473f, Feb. 2005, Linear Technology Corporation, 1630 McCarthy Blvd., Milpitas, CA 95035, United States. 
[25] Serial Peripheral Interface is a protocol on four signal lines that communicates between two digital devices.

[26] SmartFusion Customizable System-on-Chip (cSoC) Datasheet, Rev 9, Sept. 2012, Microsem Corporation, One Enterprise, Aliso Viejo CA 92656 United States.

[27] AD7451 Datasheet, C03153-0 Rev. C, Mar. 2007, Analog Devices, 3 Technology Way, Norwood, MA 02062, United States.

[28] FreeRTOS, Aavailable: http://www.freertos.org.

[29] Pretty Good Protocol is a module developed by SLAC which facilitates the bi-directional transmission of frame based messages over a two-wire physical link. 